\title{
Statistics of Electron Avalanches and Streamers
}

T. Ficker

We have studied the severe systematic deviations of populations of electron avalanches from the Furry distribution, which has been held to be the statistical law corresponding to them, and a possible explanation has been sought. A new theoretical concept based on fractal avalanche multiplication has been proposed and is shown to be a convenient candidate for explaining these deviations from Furry statistics.

Keywords: Furry and Pareto statistics, fractal multiplication of electron avalanches, fractal statistical pattern, fractal dimension.

\section{Introduction}

Electron avalanches, as precursors of stable gas discharges, have been studied from the very beginning of the 20th century. Townsend was the first to make a major study of this type of non-self-sustained discharges. In the 1930s in connection with streamer discharges, Raether [1], Loeb [2], and Meek [3] built up a new streamer theory which incorporated the Townsend electron avalanche as a necessary starting base for launching the streamer channel. According to this view, a single avalanche with a high electron population $\bar{n} \in\left(10^{8}, 10^{9}\right)$ is accompanied by UV radiation intensive enough to facilitate photoionisation as a major "driving force" of electron multiplication while collisional ionisation successively loses its governing role. According to this theory, a single big Townsend avalanche $\bar{n}>10^{8}$ starts the streamer luminous channel of cold plasma that follows the track of the initial Townsend avalanche.

At first sight it might seem that there are certain statistical similarities between streamers and Townsend avalanches. But, surprisingly, this is not the case. Both theoretical [4] and experimental results [1,5-7] have shown that electron populations $n$ of poor Townsend avalanches $\bar{n}<10^{5}$ developed inside a discharge gap of length $d$ are governed by the Furry [8] probability density $w(n, d)$

$$
\begin{aligned}
& w(n, d)=\frac{1}{\bar{n}}\left[1-\frac{1}{\bar{n}}\right]^{n-1}, \\
& \lim _{\bar{n} \rightarrow \infty} w(n, d)=\frac{1}{\bar{n}} \exp \left(-\frac{n}{\bar{n}}\right), \\
& \bar{n}=\left[\int_{0}^{x} \alpha\left(x^{\prime}\right) \mathrm{d} x^{\prime}\right]
\end{aligned}
$$

whereas populations of pre-streamer $\left(\bar{n} \in\left(10^{5}, 10^{8}\right)\right)$ and streamer $\left(\bar{n}>10^{8}\right)$ avalanches are governed by another statistical law [9-17], which is different from that of Furry. The experimental data from these highly populated avalanches is best fitted [11-17] by the Pareto distribution function

$$
w(n, d)=\text { const } \cdot n^{-(1+D)},
$$

where $D$ is the so-called fractal dimension. The different statistical behaviour of lowly and highly populated avalanches has been verified many times in our laboratory [11-17] when we have measured the height statistics of DC partial discharges with sandwiched electrode systems. Depending on the actual experimental conditions, partial discharges are in fact electron avalanches often mixed with streamers (see Fig. 1) and sometimes even developed into microscopic

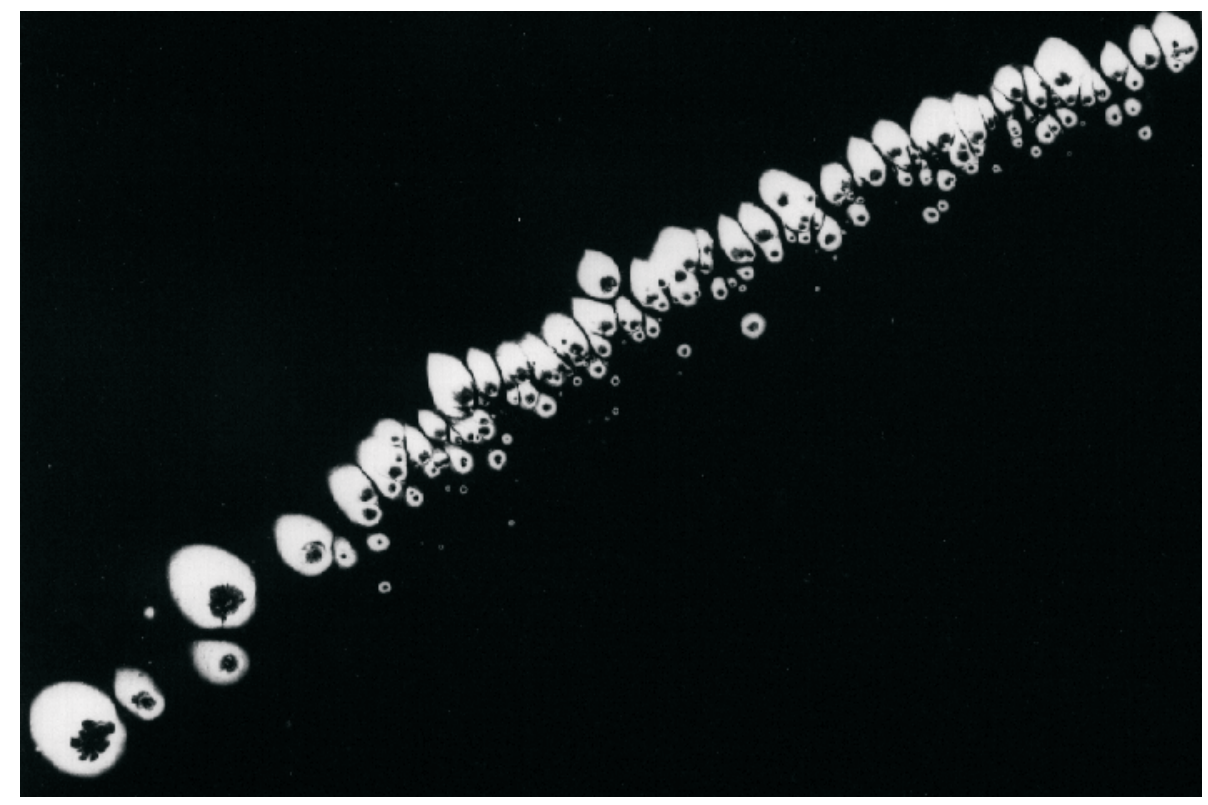

Fig. 1: Streamer spots on the dielectric barrier 


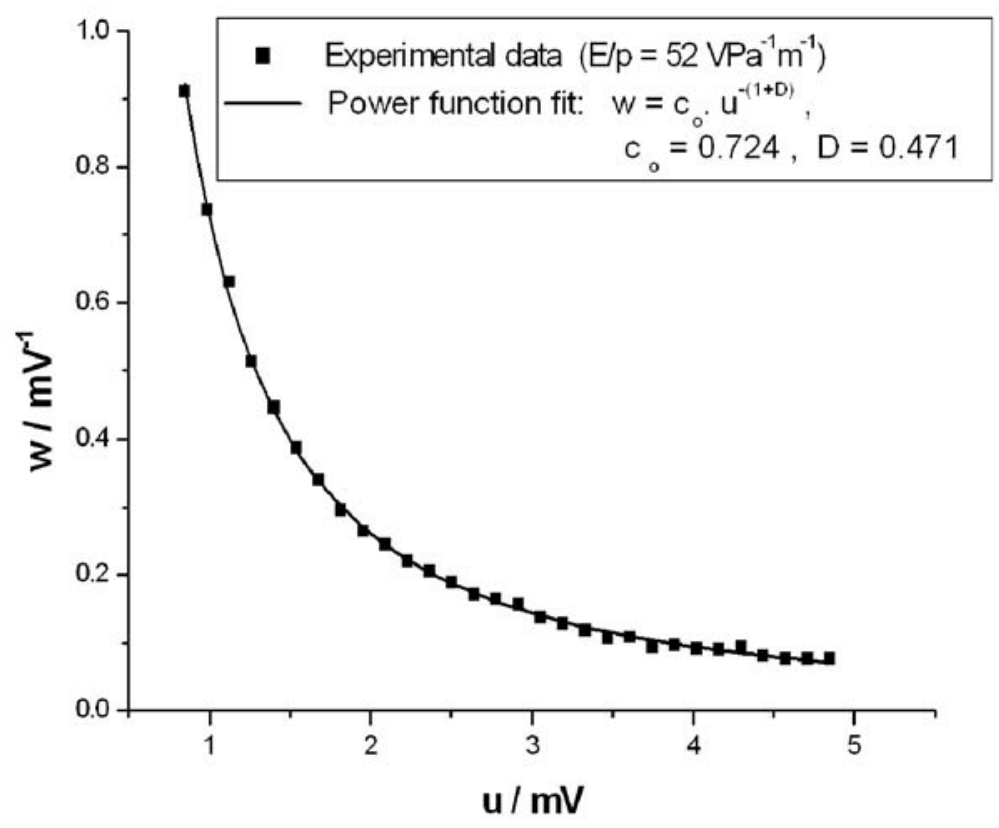

Fig. 2: Avalanche statistics $\left(E / p=52 \mathrm{~V} \mathrm{~Pa}^{-1} \mathrm{~m}^{-1}\right.$, voltage pulses) in air at normal laboratory conditions

sparks. The height statistics of DC partial discharges are nothing else than statistical distributions of electron avalanches via their populations, i.e. via the number of charge carriers that they have. Fig. 2 shows one of our avalanche statistics measured with an ultra-fast digitiser [12, 13]. Avalanches were detected across a resistance (the resistance ( $R=100 \mathrm{k} \Omega$ ) was connected in series to the discharge gap $(\mathrm{C})$ so that the two components formed a classical RC circuit - for more details see ref. [14]) as short voltage pulses with random heights $u$. Since we did not calibrate the voltage pulses $u$ against the number of electrons $n$, our resulting distribution curves $w$ are dependent on $u$ instead of $n$. Fig. 2 shows that the power function $w(u)=c_{0} \cdot u^{-(1+D)}$ represents an excellent fit of the measured data. Assuming linear proportionality $u \approx c \cdot n$, our curve $w(u)$ will preserve the same shape as that of Furry $w(n)=$ const $\cdot n^{-(1+D)}$, i.e. they will both possess the same value of the exponent $(1+D)$.

Unfortunately, no theory has been developed so far to explain the different statistical behaviour of lowly and highly populated avalanches. To resolve this puzzle, it is necessary to find the corresponding physical process underlying the phenomenon. There are several important points that should be taken into account when forming a theoretical concept explaining the cross-over from Furry statistics to Pareto statistic:

- The change in the statistical behaviour occurs simultaneously with the change in electron multiplication, i.e., when photoionisation starts dominating over collisional ionisation and highly populated avalanches appear $\left(\bar{n}>10^{5}\right)$.

- Since all fractal objects are governed by Pareto statistics, the electron multiplication mechanism that forms the Pareto set of avalanches has to be of a fractal nature, too.

- The fractal photoionisation multiplication should be based on creating additional smaller avalanches accompanying the initial (parent) avalanche, because an increase in elec- tron populations within the parent avalanches leads only to an increase in average population $\bar{n}$, which does not change the character of the Furry distribution itself (1).

A proposal for a convenient fractal mechanism of electron multiplication, capable of creating the Pareto set of electron avalanches, is formulated in the next paragraphs of this paper. In addition, a derivation of the general statistical pattern which follows the Pareto behaviour (2) together with its application to experimental data will also be presented.

\section{Fractal multiplication of electron avalanches}

On the basis of the experimental observations and deductions mentioned above, it is clear that the multiplication mechanism of highly populated avalanches, whose populations follow Pareto's distribution, has to be governed by a fractal scenario that should be very similar to the following:

(i) Beside a parent avalanche a series of additional smaller avalanches arises inside the discharge gap. These smaller avalanches are generated in a hierarchical manner with different mean populations $\bar{n}_{d}$ to fulfil the fractal scenario

$$
\left\{\bar{n}_{d}\right\}_{j=0}^{J}=\left\{\mathrm{e}^{\alpha(d-j \bar{\Delta})}\right\}_{j=0}^{J}, \quad J \leq \frac{d}{\Delta}-1 .
$$

In this way the number of less populated avalanches increases and, as a consequence, deviations from the Furry distribution may occur.

(ii) Multiplication of highly populated avalanches with mean populations $\left\{\bar{n}_{d}\right\}_{j=0}^{j}$ must be generated according to a fractal scenario of branching or partitioning like most fractals when going to smaller scales. Therefore, some type of fractal avalanche branching should be taken into account. The branching should originate 
with a parent avalanche possessing a mean population $n_{d, 0}=\mathrm{e}^{\alpha d}$ (fractal initiator). After passing a certain initial distance $\bar{\Delta}>0$ and gathering a certain number of energetic electrons $\bar{N}=\mathrm{e}^{\alpha d} \geq 1$, which are capable of creating a group of UV photons, a photoionisation process starts and a swarm of $\bar{K} \geq 1$ smaller avalanches with mean populations $\bar{n}_{d, 1}=\mathrm{e}^{\alpha(d-\bar{\Delta})}$ appear along the parent avalanche. Let us call them "side avalanches of the first generation". Thus the side avalanches of the first generation actually represent the so-called fractal generator given by multiplicity $\bar{K}$. The side avalanches, once created, become parent avalanches for the next generation of new side avalanches. So, the side avalanches of the first generation become parent avalanches for the side avalanches of the second fractal generation with the mean population $\bar{n}_{d, 2}=\mathrm{e}^{\alpha(d-2 \bar{\Delta})}$. This process of avalanche multiplication may or may not continue up to the last possible generation $J=d / \bar{\Delta}-1$ with the mean population $\bar{n}_{d, J}=\mathrm{e}^{\alpha(d-J \cdot \bar{\Delta})}$. Provided the multiplying process reaches the $\mathrm{j}$-th generation, the mean (average) total number of side avalanches in this generation is just $\bar{K}^{J}$. The described multiplicative process yields a hierarchy of avalanches and when extended to infinity $(J \rightarrow \infty)$, it yields an infinite set of avalanches that is similar to the well-known fractal object called the Cantor fractal set [18]. Using this similarity, a relation between avalanche characteristics and properties of the Cantor fractal set can easily be found

$\bar{K}^{j}=\left(\frac{L_{o}}{l_{j}}\right)^{D}=\left(\frac{\bar{n}_{d, o}}{\bar{n}_{d, j}}\right)^{D} \Rightarrow \bar{K}^{j}=\left(\bar{N}^{j}\right)^{D} \Rightarrow D=\frac{\ln \bar{K}}{\ln \bar{N}}$.

Since all the fractal objects obey the Pareto statistics with probability density in the form of the power law (2), the studied avalanche set, being of a fractal nature, will also follow this statistical law

$w(n, x)=$ const $\cdot n^{-(1+D)}=$ const $\cdot n^{-\left(1+\frac{\ln \bar{K}}{\ln \bar{N}}\right)}$.

Such a strictly deterministic mechanism, which has already been described, might hardly be expected in a real situation. Instead, a strongly stochastic mechanism is more probable with certain distributions of the quantities $\Delta, K$, and $N$. However, the use of their average values $\bar{\Delta}, \bar{K}$, and $\bar{N}$ makes the treatment more realistic and partly advocates the deterministic view of the problem.

(iii) The described fractal mechanism of multiplication of highly populated avalanches anticipates that the most probable place where a parent avalanche initiates side avalanches is in some of the first $\Delta$-intervals because, due to diffusion at a larger distance, the parent avalanche is broadened enough to integrate the side avalanches.

The foregoing paragraphs have summarised the main properties of the concept of fractal multiplication of highly populated avalanches. If all these assumptions are sound, the derivation of a new statistical pattern using the existence of side avalanches should be fruitful. Naturally, this new pattern must be capable of generating linear behaviour in the bilogarithmic co-ordinates with the slope $-(1+\ln \bar{K} / \ln \bar{N})$. Such a derivation is realised in the next section.

\section{Statistical pattern of fractal avalanche multiplication}

With reference to points (i)-(iii), the probability densities for generations of side avalanches can be formed as follows

$\mathbf{i = 0 :}$ Zero generation - parent avalanche

$$
w_{0}(n, d)=\frac{1}{\bar{n}_{d}}\left[1-\frac{1}{\bar{n}_{d}}\right]^{n-1} .
$$

$\mathbf{j}=1$ : The first generation of side avalanches

$$
w_{1}(n, d-\bar{\Delta})=\frac{\bar{K}}{\bar{n}_{d-\bar{\Delta}}}\left[1-\frac{1}{\bar{n}_{d-\bar{\Delta}}}\right]^{n-1} .
$$

$\mathbf{j = 2 :}$ The second generation of side avalanches

$$
w_{2}(n, d-2 \bar{\Delta})=\frac{\bar{K}^{2}}{\bar{n}_{d-2 \bar{\Delta}}}\left[1-\frac{1}{\bar{n}_{d-2 \bar{\Delta}}}\right]^{n-1} .
$$

j: $\quad$ The $j$-th generation of side avalanches

$$
\begin{aligned}
w_{2}(n, d-j \bar{\Delta}) & =\frac{\bar{K}^{j}}{\bar{n}_{d-j \cdot \bar{\Delta}}}\left[1-\frac{1}{\bar{n}_{d-j \cdot \bar{\Delta}}}\right]^{n-1} \\
& =\frac{(\bar{K} \cdot \bar{N})^{j}}{\bar{n}_{d}}\left[1-\frac{\bar{N}^{j}}{\bar{n}_{d}}\right]^{n-1},
\end{aligned}
$$

where

$$
\begin{gathered}
\bar{n}_{d-j \cdot \bar{\Delta}}=\mathrm{e}^{\alpha(d-j \cdot \bar{\Delta})}=\bar{n}_{d} \cdot\left(\mathrm{e}^{\alpha \cdot \Delta}\right)^{-j}=\frac{\bar{n}_{d}}{\bar{N}^{j}}, \quad \bar{N}=\mathrm{e}^{\alpha \cdot \Delta}, \\
\vdots \\
\ldots \rightarrow \infty \ldots
\end{gathered}
$$

The probability density $F(n, d)$ measured at the anode, which is placed at a distance $d$ from the cathode, is given as the sum of all the probability densities of the avalanches created within the discharge gap

$$
\begin{aligned}
F(n, d) & =\sum_{j=0}^{J} w_{j}(n, d-j \cdot \bar{\Delta}) \\
& =\frac{1}{\bar{n}_{d}} \sum_{j=0}^{J}(\bar{K} \cdot \bar{N})^{j} \cdot\left[1-\frac{\bar{N}^{j}}{\bar{n}_{d}}\right]^{n-1},
\end{aligned}
$$

where

$$
J=\frac{d}{\bar{\Delta}}-1, \quad \bar{N} \geq 1, \quad \bar{K} \geq 1 .
$$

Within the exponential approximation (1) the total probability density (11) reads

$F(n, d) \approx \frac{1}{\bar{n}_{d}} \sum_{j=0}^{J}(\bar{K} \cdot \bar{N})^{j} \cdot \exp \left[-\frac{n \cdot \bar{N}^{j}}{\bar{n}_{d}}\right]$.

Relations (11) and (13) are generalised statistical distributions that include the original Furry distribution (1) and its exponential approximation as special cases $(j=0)$ when no side avalanches are generated.

At first sight the probability densities (11) / (13) seem unlikely to follow the Pareto power law (2), but the opposite is 


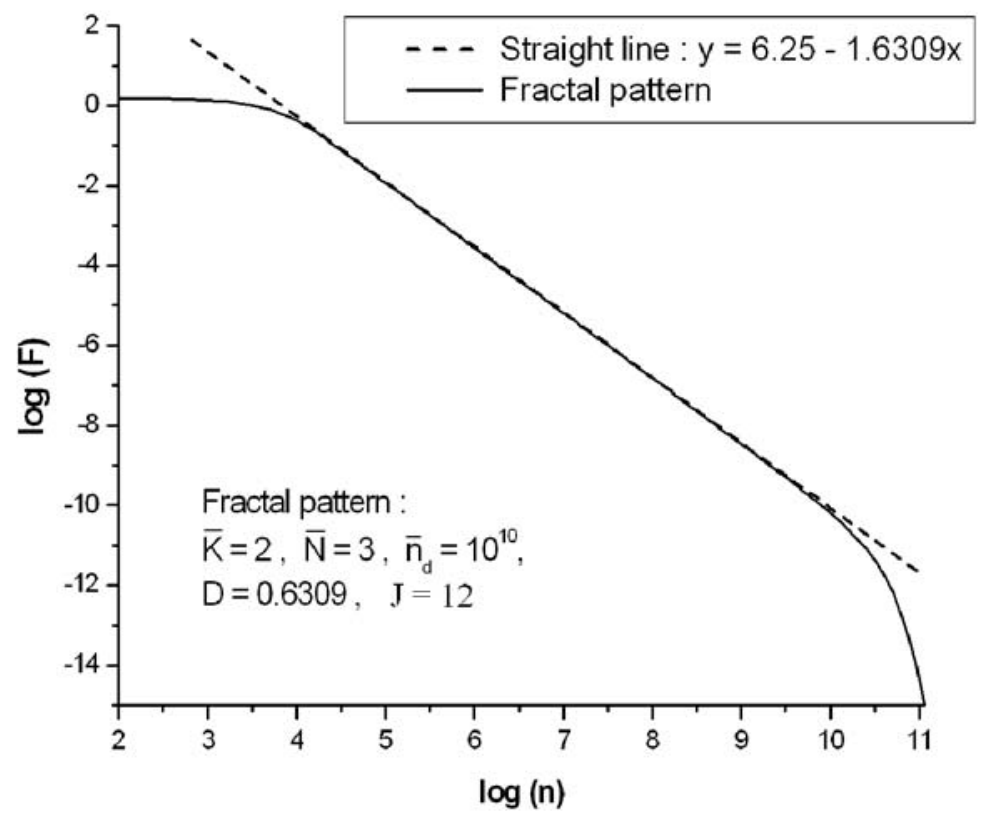

Fig. 3: Fractal statistical pattern developed into a power law

true. When these functions are plotted with convenient parameters $\bar{n}_{d}, \bar{K}$, and $\bar{N}$ within bilogarithmic co-ordinate systems, their graphs indeed show linear sections (power behaviours) spanning over many orders of magnitudes of electron populations - see Fig. 3. The relation for the slope

$$
s=-\left(1+\frac{\ln \bar{K}}{\ln \bar{N}}\right)=-1.6309
$$

is also fulfilled, as can be verified from Fig. 3. Nevertheless, to provide full rigorous grounds for the fractality (power law behaviour) of the generalised probability density $F(n, d)$, it would be necessary to perform a full mathematical proof of this property. This would not be an original task, as several researchers [19-28] have worked on a similar problem, though with different starting functions (usually of Lévy type).
Professor T. F. Nonnenmacher - inspired probably by theoretical works [19], [23] dealing with the origin of the fractal scaling laws in biophysics - has systematically studied [24-28] a similar problem that emerged from protein gating kinetics. As a prominent mathematical physicist he succeeded in finding an original way to transform the functions that have assumed the form of a discrete exponential chain, like series (13), into the power law functions. The required exact mathematical proof can therefore be found in his works [24-28].

Finally, to illustrate straightforwardly the capability of the generalised statistical pattern (17) of Furry's functions to provide a faithful fit for experimental data of population statistics, the experimental data from Fig. 3 has been fitted by pattern (17) in bilogarithmic co-ordinates - see Fig. 4. As can be seen from the two figures, the fractal dimensions are in excel-

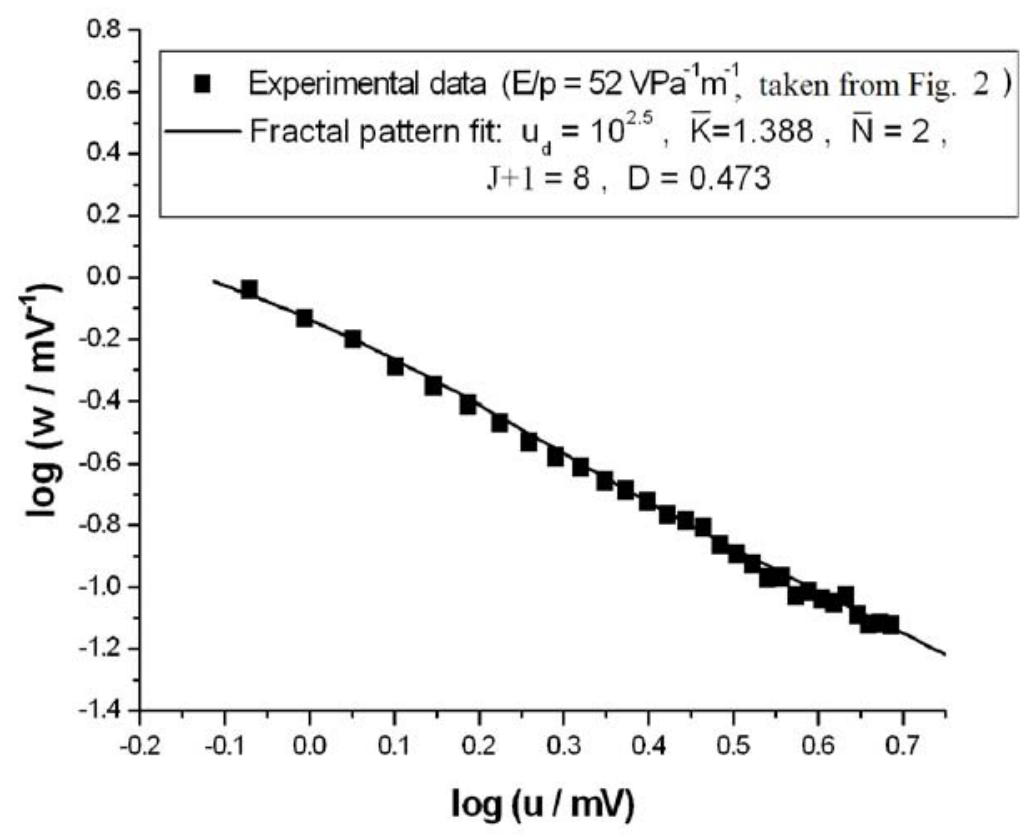

Fig. 4: Fractal pattern fit of pre-streamer statistics (voltage pulses - data taken from Fig. 2) 
lent agreement $(\approx 0.471$ versus $\approx 0.473)$. At this point we would like to mention that all other data measured in our laboratory has been processed in the same way, showing the clear capability of the statistical pattern (17) to reproduce faithfully statistical data of highly populated electron avalanches. In brief, the generalised statistical patterns (17) seem to provide very convenient approximations capable of incorporating all the main specific features of fractal avalanche multiplication.

\section{Conclusions}

In conclusion we would like to underline several main points that have been presented in this paper:

- A new concept of fractal multiplication of highly populated avalanches (streamer-like avalanches) has been proposed. The concept is based on a generalised photoionisation mechanism leading to side branching of avalanches.

- The branching may propagate to higher generations of side avalanches. This process is inherently stochastic and requires the introduction of average multiplicity $\bar{K}$ and average number $\bar{N}$ of initiating electrons to provide an analytical description of the branching procedure.

- The generalised statistical pattern (17) representing the probability density of avalanches created in a discharge gap has been derived. This pattern includes the original Furry distribution (1) and its exponential approximation as special cases $(J=0)$ when no side avalanches are generated.

- The capability of the generalised statistical pattern (17) to fit faithfully experimental data from avalanche experiments has also been illustrated (Fig. 4).

\section{Acknowledgments}

This work has been supported by the Grant Agency of the Czech Republic under Grant No. 202/07/1207.

\section{References}

[1] Raether, H.: Electron Avalanches and Breakdown in Gases. London: Butterworths, 1964.

[2] Loeb, L. B.: Basic Processes of Gaseous Electronics. Berkley CA: University of California Press, 1960.

[3] Meek, J. M., Craggs, J. D.: Electrical Breakdown of Gases. New York: Willey, 1978.

[4] Wijsman R.: Phys. Rev. Vol. 75 (1949), p. 833.

[5] Van Brunt, R. J.: IEEE Trans. El. Insul. Vol. 26 (1991), p. 902.

[6] Frommhold, L.: Zeitschrift für Physik, Vol. 150 (1958), p. 172.

[7] Schlumbohm, H.: Zeitschrift für Physik, Vol. 152 (1958), p. 49.

[8] Furry, W. H.: Phys. Rev. Vol. 52 (1937), p. 569.

[9] Schlumbohm, H.: Zeitschrift für Physik, Vol. 151 (1958), p. 563.
[10] Richter, K.: Zeitschrift für Physik, Vol. 158 (1960), p. 312.

[11] Ficker, T.: J. Appl. Phys. Vol. 78 (1995), p. 5289.

[12] Ficker, T., Macur, J., Kliment, M., Filip, S., Pazdera, L.: J. El. Eng. Vol. 51 (2000), p. 240.

[13] Ficker, T, Macur, J., Pazdera, L., Kliment, M., Filip, S.: IEEE Trans. Diel. El. Insul. Vol. 8 (2001), p. 220.

[14] Ficker, T.: IEEE Trans. Diel. El. Insul. Vol. 10 (2003), p. 689 .

[15] Ficker, T.: IEEE Trans. Diel. El. Insul. Vol. 10 (2003), p. 700 .

[16] Ficker, T., Macur, J., Kapička, V.: Czech. J. Phys. Vol. 53 (2003), p. 509.

[17] Ficker, T.: IEEE Trans. Diel. El. Insul. Vol. 11 (2004), p. 136.

[18] Mandelbrot, B. B.: The Fractal Geometry of Nature. New York: Freeman, 1983.

[19] Montroll, E. W., Shlesiger, M. F.: Proc. Natl. Acad. Sci. Vol. 79 (1982), p. 3380.

[20] Shlesiger, M. F.: J. Stat. Phys. Vol. 36 (1984), p. 639.

[21] Montroll, E. W., Bendler, J. T.: J. Stat. Phys. Vol. 34 (1984), p. 129.

[22] Shlesinger, M. F., West, B. J., Klafter, J.: Phys. Rev. Lett. Vol. 50 (1988), p. 1100.

[23] West, B. J., Bhargava, V., Goldberger, A. L.: J. Appl. Physiol. Vol. 60 (1988), p. 1089.

[24] Nonnenmacher, T. F., Nonnenmacher, D. J. F.: In: Albeverio. S., Casati, G., Cattaneo, U., Merlini, D., Moresi R. (eds.): Stochastic processes, Physics and Geometry. Proc. of the $2^{\text {nd }}$ Ascona/Locarno Conference: World Scientific, July 1988.

[25] Nonnenmacher, T. F., Nonnenmacher, D. J. F.: Phys. Lett. A, Vol. 140 (1989), p. 323.

[26] Nonnenmacher, T. F.: Coll. Polym. Sci. Vol. 267 (1989), p. 753.

[27] Nonnenmacher, T. F.: Coll. Polym. Sci., Vol. 268 (1990), p. 401.

[28] Nonnenmacher, T. F., Losa, G. A., Weibel, E. R. (eds.): Fractals in Biology and Medicine. Basel: Birkhäser Verlag, 1994.

Prof. RNDr. Tomáš Ficker, DrSc.

phone: +420541 147661

e-mail: ficker.t@fce.vutbr.cz

Department of Physics

University of Technology

Faculty of Civil Engineering

Žižkova 17

66237 Brno, Czech Republic 